\title{
Comportement dissymétrique des dislocations entre traction et compression dans des superalliages base-nickel
}

\author{
Brigitte Décamps $\left({ }^{1}\right)$, Marc Condat $\left({ }^{1}\right)$ et Allan J. Morton $\left({ }^{2}\right)$ \\ ( $\left.{ }^{1}\right)$ CNRS, Laboratoire de métallurgie Structurale, Université Paris-Sud, Bât. 413, 91405 Orsay \\ Cedex, France \\ ( ${ }^{2}$ ) CSIRO, Division of Materials Science and Technology, Locked Bag 33, Clayton Vic. 3168, \\ Australia
}

(Reçu le 4 juin 1991, accepté le 31 janvier 1992)

\begin{abstract}
Résumé. - La microscopie électronique en transmission a été utilisée pour étudier les configurations élémentaires de cisaillement des précipités $\gamma^{\prime}$ dans des superalliages base nickel après des essais de fluage et des essais dynamiques. Un changement de configuration a été observé entre les essais de traction et de compression. Cette assymétrie traction/compression peut être expliquée en considérant la résultante de deux effets: le franchissement de l'interface par des partielles de Shockley et l'effet de la contrainte appliquée sur la largeur de dissociation des dislocations de matrice. Un modèle original de cisaillement des précipités par des dislocations de matrice a été proposé ainsi qu'une explication de la dépendence vis à vis de la température des processus de cisaillement.
\end{abstract}

\begin{abstract}
The elementary shearing configurations of $\gamma^{\prime}$ precipitates in single crystals of Nibased superalloys have been studied after creep and dynamical experiments using TEM techniques. A change of configurations has been observed between tension and compression. This tension/ compression asymetry may be explained by considering the interplay between the crossing of the $\gamma / \gamma^{\prime}$ interface by elementary Shockley dislocations and the effect of the applied stress on the dissociation width of matrix dislocations. An original model of shear of precipitates by dissociated matrix dislocations is proposed and an understanding of the dependence of the shearing processes with the temperature is provided.
\end{abstract}

\section{Introduction.}

Le cisaillement des précipités $\gamma^{\prime}$ joue un rôle important dans les processus de déformation des superalliages à forte fraction volumique de précipités. Il est bien connu que ces processus de déformation dépendent de la température [1-3]. Le cisaillement des précipités impliquant des superdéfauts d'empilement (SSF) est un des modes importants de déformation. Pendant de nombreuses années, un modèle dit de "Kear" [4] a servi de référence pour expliquer la formation de ces SSF. Ce n'est que très récemment, avec l'utilisation de la microscopie électronique en champ 
faible [5-8], que la validité de ce modèle a été remise en question: les dislocations de matrice appartenant aux configurations de cisaillement sont $a / 2<110>$ et non pas $a / 2<112>$.

L'objet de cette publication est de présenter une synthèse des observations qui nous ont amenées à proposer un mécanisme original de cisaillement des précipités par une simple dislocation de matrice $a / 2<110>$ dissociée avec nucléation d'une dislocation de Shockley pour former la SSF.

Sur la base de ce modele, des corrélations entre comportement à l'échelle microscopique et comportement à l'échelle macroscopique ont été proposées.

\section{Matériaux et techniques expérimentales.}

Des essais mécaniques ont été effectués sur des monocristaux de superalliages base nickel denommés AM1 (Ni- 6,5Co, 7,5Cr, 5,3Al, 5,5W, 8Ta, 2Mo, 1,2Ti), fourni par la société SNECMA, et AM3 (Ni- 5Co, 6,5Cr, 6Al, 4,5W, 4,2Ta, 2,3Mo, 2Ti, 0,5V), fourni par l'ONERA.Pour ces alliages, les précipités de structure $\mathrm{L1}_{2}$, après un traitement de mise en solution et de précipitation, sont cubiques et d'une taille moyenne de $500 \mathrm{~nm}$ pour une fraction volumique voisine de $65 \%$.

Les essais mécaniques effectués sont les suivants:

- fluage traction sur AM1 (axe <001>) à $760^{\circ} \mathrm{C}$ sous $730 \mathrm{MPa}$ arrêté à $0,4 \%$ de déformation plastique;

- fluage traction sur AM3 (axe <001>) a $760^{\circ} \mathrm{C}$ sous $750 \mathrm{MPa}$ arrêté à $0,49 \%$ de déformation plastique (temps de l'essai: $22 \mathrm{~h}$ );

- fluage traction sur AM1 (axe voisin de <011>) à $950^{\circ} \mathrm{C}$ sous $270 \mathrm{MPa}$ arrêté à $0,4 \%$ de déformation plastique;

- compression dynamique sur AM1 (axe <001>) à $950^{\circ} \mathrm{C}$ et arrêté à $0,4 \%$ de déformation plastique $(V=0,05 \mathrm{~mm} / \mathrm{mn})$;

- fluage compression sur AM3 (axe <001>) à température intermédiaire $\left(750^{\circ} \mathrm{C}\right.$ et $810^{\circ} \mathrm{C}$ ) sous respectivement 680 et $650 \mathrm{Mpa}$ et arretés à 0,5 et $0,4 \%$ de déformation plastique (temps des essais : $7 \mathrm{~h} 23 \mathrm{mn}$ et $1 \mathrm{~h}$ );

- traction dynamique sur AM1 (axe <001>) à $950^{\circ} \mathrm{C}$ et arrêté à $1,4 \%$ de déformation $(990 \mathrm{MPa})$.

Les échantillons sont découpés parallèlement à des plans $\{111\}$. Les observations sont faites à l'aide d'un microscope Jeol 2000EX en utilisant les techniques du champ faible [9] et du champ clair [10]. Tout au long de cette étude, les vecteurs de Burgers des dislocations sont définis selon la convention FS/RH [11] et en utilisant le tétraèdre de Thompson [12].

\section{Résultats expérimentaux.}

3.1 FluAge TRACTION À TEMPÉRATURE INTERMÉdiAire $\left(\approx 760^{\circ} \mathrm{C}\right)[7,13]$. — Dans les deux alliages AM1 et AM3, les observations montrent que les précipités sont cisaillés par des dislocations séparées par des défauts d'empilement de superréseau (SSF) qui sont en majorité intrinsèques (SISF). Les configurations de cisaillement font intervenir des dislocations de matrice $a / 2<110>$ qui laissent à l'interface des dislocations de Shockley de nature différente de celles qui cisaillent les précipités.

La figure 1a représente une micrographie en champ faible caractéristique de l'échantillon d'AM1 déformé en fluage traction à $760^{\circ} \mathrm{C}$. La configuration complète a été analysée à l'aide d'autres réflections non présentées ici et un schéma de la configuration est donné en figure $1 b$. Les dislocations de matrice BD et BC qui participent au cisaillement sont écartées l'une de l'autre 

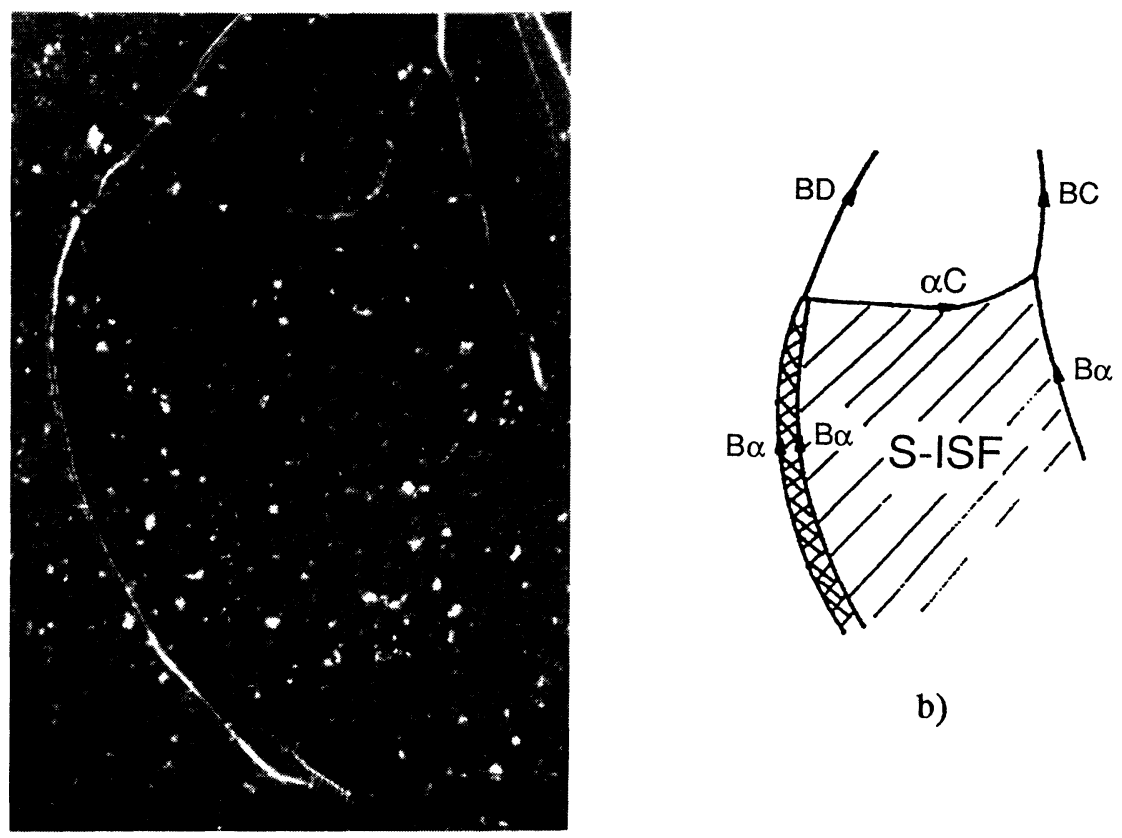

b)

a)

Fig. 1. - Configuration de cisaillement d'un précipité $\gamma^{\prime}$ par un couple de dislocations de matrice BD/BC dans l'alliage $\mathrm{AM} 1$, déformé en fluage traction à $760^{\circ} \mathrm{C}$. (a) Microscopie en champ faible, plan (111), $g=\overline{2} 20$ (marque $=0,05 \mu \mathrm{m}$ ). (b) Représentation schématique de la configuration, S-ISF (SSF intrinsèque).

[Shearing configuration of a $\gamma^{\prime}$ precipitate by a pair of matrix dislocations BD/BC in the alloy AM1 deformed in tension creep at $760^{\circ} \mathrm{C}$. (a) Weak-beam micrograph, (111)plane, $g=\overline{2} 20$ (bar $=0.05 \mu \mathrm{m}$ ). (b) Scheme of the configuration, S-ISF (superlattice intrinsic stacking fault).]

et connectées par une Shockley $\alpha \mathrm{C}$ à l'interface $\gamma / \gamma^{\prime}$ de nature différente des Shockley qui cisaillent le précipité. La dislocation $2 \mathrm{~B} \alpha$ qui cisaille le précipié est dissociée en $\mathrm{B} \alpha+\mathrm{B} \alpha$ séparées par un défaut complexe dont l'énergie est voisine de $80 \mathrm{~mJ} / \mathrm{m}^{2}$ (théorie élastique isotrope). La dislocation $\mathrm{B} \alpha$ à droite de la configuration reste bloquee à l'interface $\gamma / \gamma^{\prime}$. Le caractère intrinsèque du défaut d'empilement a été déterminé par une analyse du sens du vecteur de Burgers de la dislocation $2 \mathrm{~B} \alpha$ en champ clair et en champ faible.

Des configurations similaires ont été observées dans l'alliage AM3 déformé en fluage traction à $760^{\circ} \mathrm{C}$. L'énergie de défaut complexe est trouvée du même ordre de grandeur que pour l'AM1 $\left(\approx 70 \mathrm{~mJ} / \mathrm{m}^{2}\right)$.

La figure 2a présente un autre type de cisaillement où les deux dislocations de matrice participant au cisaillement ont même vecteur de Burgers. Un schéma de la configuration est donné en figure $2 \mathrm{~b}$. Comme précédemment, les dislocations de matrice sont connectées par une Shockley à l'interface $\gamma / \gamma^{\prime}$ et les SuperShockley $(a / 3\langle 112\rangle)$ qui cisaillent le précipité sont dissociées (énergie de défaut complexe de l'ordre de $80 \mathrm{~mJ} / \mathrm{m}^{2}$ ).

3.2 FLUAGE TRACTION À $950^{\circ} \mathrm{C}$ [14]. - Dans cet échantillon, les configurations observées ne font pas intervenir de cisaillement par des dislocations séparées par des superdéfauts d'empilement (SSF). 

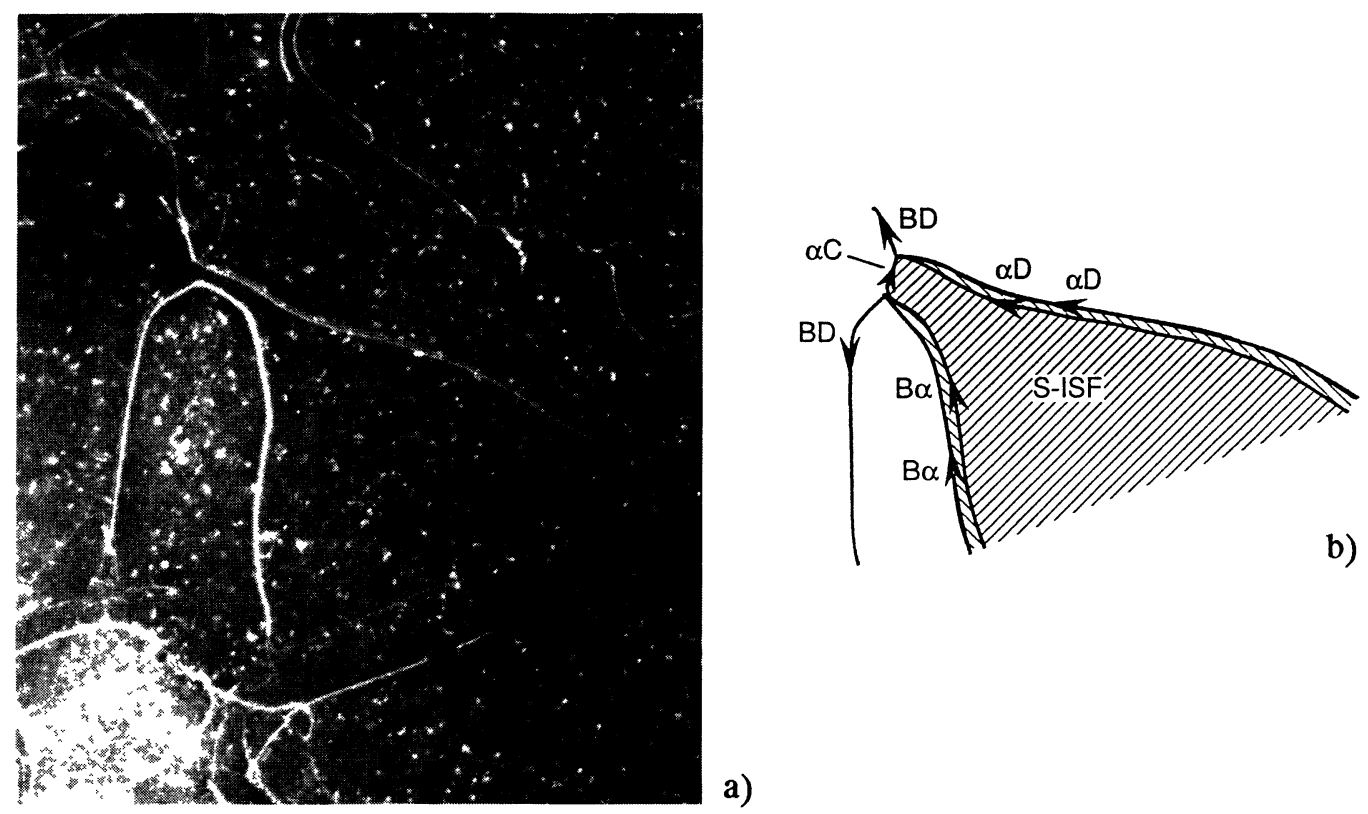

a)

Fig. 2. - Configuration de cisaillement d'un précipité $\gamma^{\prime}$ par un couple de dislocations de matrice BD dans l'alliage AM1 déformé en fluage traction à $760^{\circ} \mathrm{C}$. (a) Micrographie en champ faible, plan (111), $g=20 \overline{2}$ (marque $=0,08 \mu \mathrm{m}$ ). (b) Représentation schématique de la configuration, S-ISF (SSF intrinsèque).

[Shearing configuration of a $\gamma^{\prime}$ precipitate by a pair of matrix dislocations BD in the alloy AM1 deformed in tension creep at $760^{\circ} \mathrm{C}$. (a) weak-beam micrograph, (111) plane, $g=20 \overline{2}$ (bar $=0.08 \mu \mathrm{m}$ ). (b) Scheme of the configurartion, S-ISF (superlattice intrinsic stacking fault).]

La figure 3 présente deux micrographies en champ faible montrant deux dislocations $a / 2$ [110] dissociées en deux partielles de Shockley dans le plan (111) de la lame. Les deux partielles sont visibles pour les deux réflections de la figure 3 tandis que le défaut d'empilement est hors contraste. L'analyse basée sur d'autres réflections montre que le défaut d'empilement est intrinsèque dans la matrice et il n'y a nulle part inversion des partielles tout au long des dislocations $\left(\mathrm{bp}_{1}=\right.$ $a / 6[\overline{1} 2 \overline{1}]$ et $\left.\mathrm{bp}_{2}=a / 6[\overline{2} 11]\right)$.

Deux phénomènes sont présents à l'interface $\gamma / \gamma^{\prime}$ :

- Montée des dislocations sous forme de constrictions sur les dislocations de matrice (partie B) ainsi que l'ont montré Décamps et Condat [6].

- Transformation progressive d'un défaut intrinsèque de matrice en défaut complexe (parties C et D). Les valeurs calculées des énergies de défaut sont de l'ordre de $80 \mathrm{~mJ} / \mathrm{m}^{2}$ pour la partie C et $45 \mathrm{~mJ} / \mathrm{m}^{2}$ pour la partie $\mathrm{D}$ (théorie élastique isotrope). Il a été montré que la coalescence des précipités était responsable de cette transformation [14]: les dislocations de matrice entrent dans le précipité plutôt que d'être repoussées dans la matrice.

3.3 COMPRESSION DYNAMIQUE À $950^{\circ} \mathrm{C}$ [13]. - Les précipités sont cisaillés principalement par des dislocations séparées par des superdéfauts d'empilement (SSF) de nature extrinsèque (S-ESF). Les analyses montrent que les processus de cisaillement font intervenir des dislocations de matrice laissant à l'interface une Shockley de nature différente de celles qui cisaillent le précipité. Les configurations observées sont du même type que celles rencontrées en fluage traction à température intermédiaire mis à part la nature des SSF. 

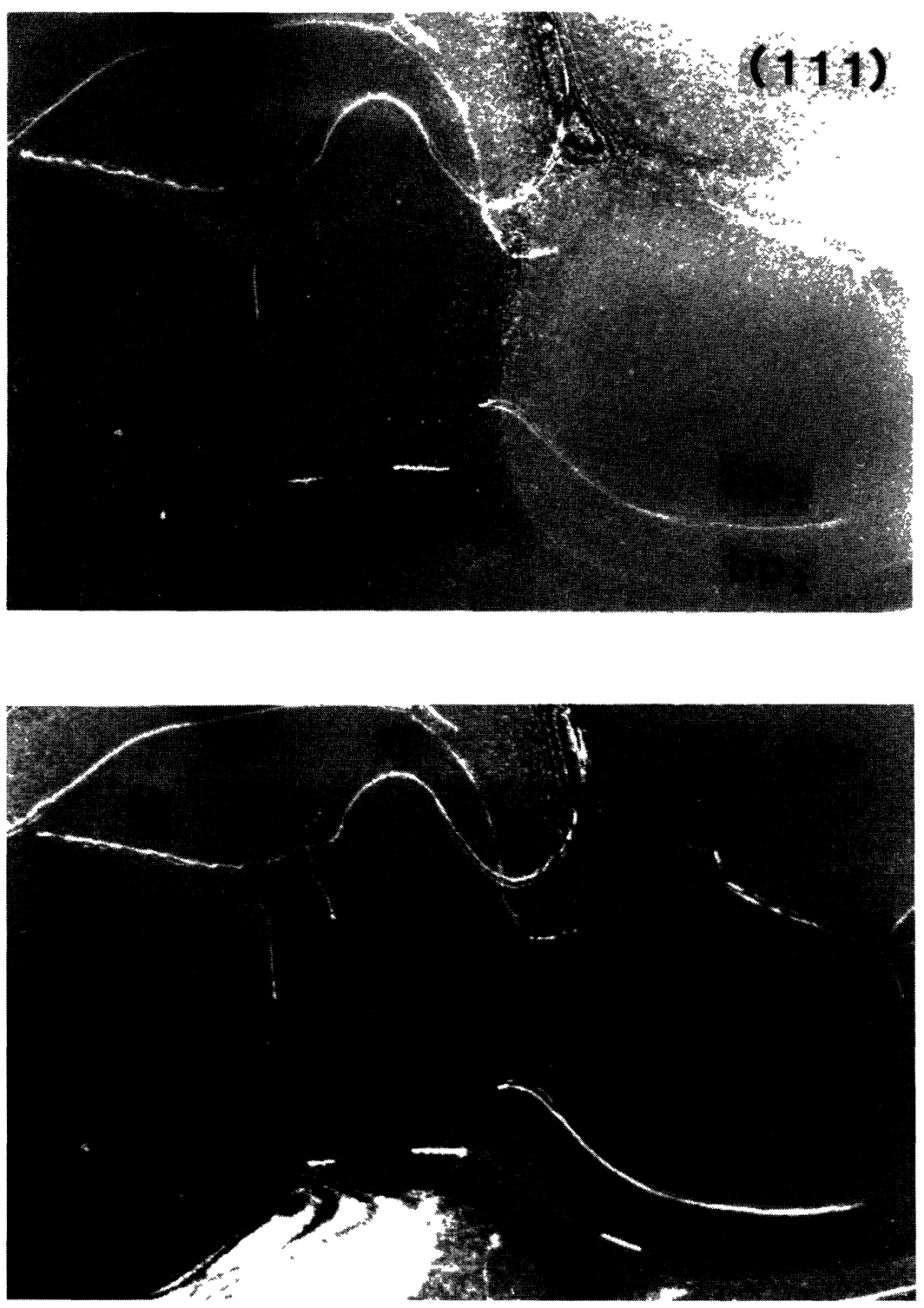

Fig. 3. - Dislocations $a / 2<110>$ situées à l'interface $\gamma / \gamma^{\prime}$ dans l'alliage AM1 déformé en fluage traction à $950^{\circ} \mathrm{C}$ : (a) micrographie en champ faible, $g=2 \overline{2} 0$; (b) Micrographie en champ faible, $g=\overline{2} 20$.

$\left[a / 2<110>\right.$ dislocations located at the $\gamma / \gamma^{\prime}$ interface in the alloy AM1 deformed in tension creep at $950^{\circ} \mathrm{C}$ : (a) weak-beam micrograph in $g=2 \overline{2} 0$. (b) weak-beam micrograph in $g=\overline{2} 20$.

La figure 4 présente une configuration typique de cisaillement. Pour la figure $4 a$, le défaut d'empilement est visible tandis que pour la figure $4 \mathrm{~b}$, il est hors contraste. L'analyse totale de la configuration a été faite en utilisant d'autres réflections et un schéma en est donné en figure 4c. On peut remarquer dans cette configuration que le cisaillement apparait avoir été initié par une dislocation de matrice unique (voir la partie haute de la configuration Fig. 4). Ainsi que 
précédemment, les dislocations qui cisaillent le précipité sont dissociées $(2 \alpha \mathrm{B}=\alpha \mathrm{B}+\alpha \mathrm{B})$ et l'énergie de défaut compléxe a été trouvée voisine de $80 \mathrm{~mJ} / \mathrm{m}^{2}$ (théorie élastique isotrope).
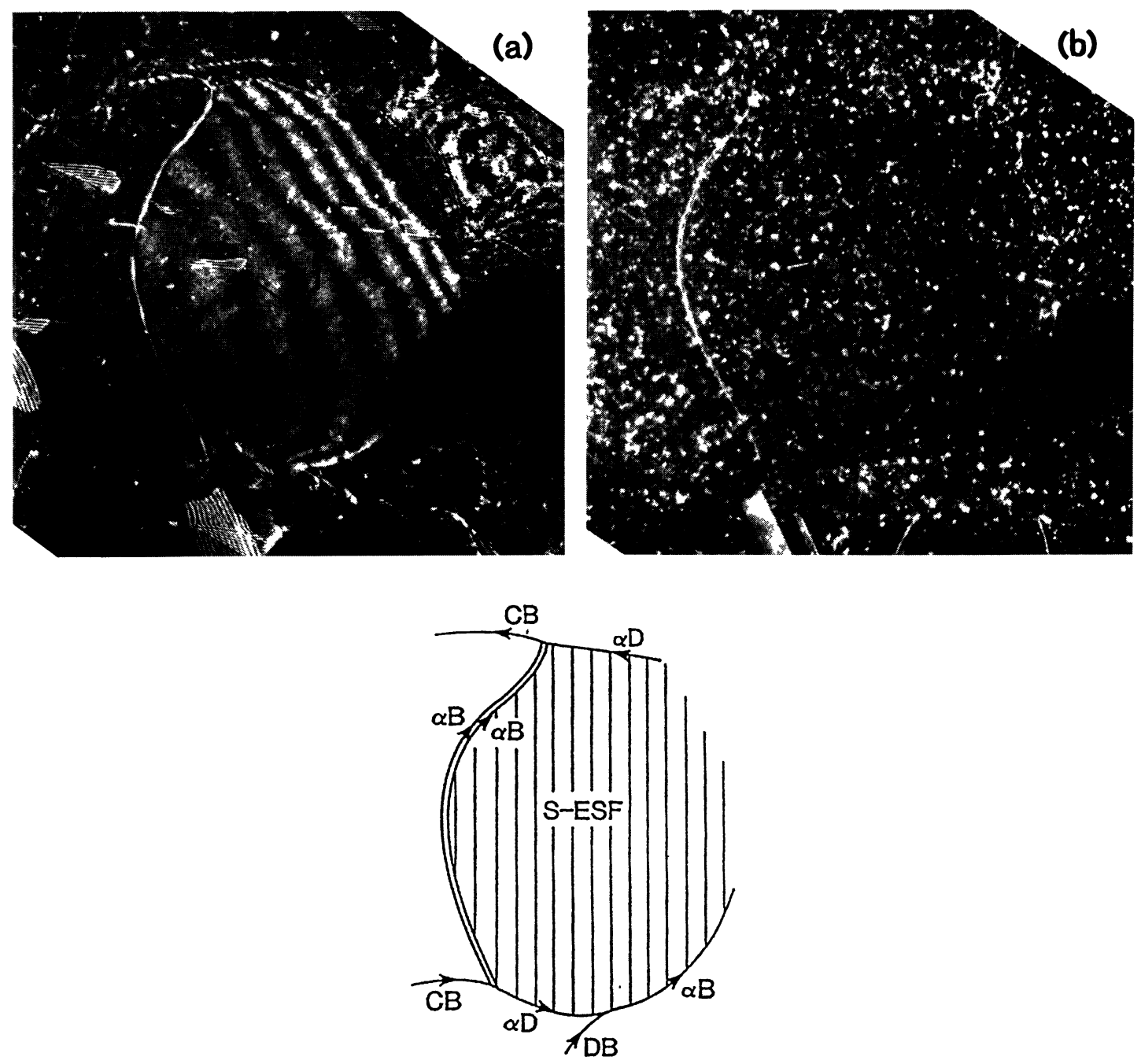

c)

Fig. 4. - Configuration de cisaillement d'un précipité $\gamma^{\prime}$ dans l'alliage AM1 déformé en compression dynamique à $950^{\circ} \mathrm{C}$. (a) micrographie en champ faible, $g=\overline{1} 11$; (b) micrographie en champ faible, $g=\overline{2} 02$; (c) représentation schématique de la configuration, S-ESF (SSF extrinsèque).

[Shearing configuration of a $\gamma^{\prime}$ precipitate in the alloy AM1 deformed in dynamical compression at $950^{\circ} \mathrm{C}$ : (a) weak-beam micrograph in $g=\overline{1} 11$; (b) weak-beam micrograph in $g=202$; (c) Scheme of the configuration, S-ESF (superlattice extrinsic stacking fault).]

3.4 TRACTION DYNAMIQUE À $950^{\circ} \mathrm{C}$ [13]. - Les observations montrent que les précipités sont cisaillés par des superdéfauts d'empilement (SSF). Les analyses faites comme précédemment sur des défauts situés dans le plan (111) de la lame et dans des plans $\{111\}$ inclinés révèlent 
un mélange de SSF intrinsèques et extrinsèques avec une proportion apparente plus élevée de défauts intrinsèques.

La figure 5 présente une configuration typique révélant la présence des deux types de défauts dans une même zone de l'échantillon. Un schéma de la configuration est donné figure $5 \mathrm{~d}$.
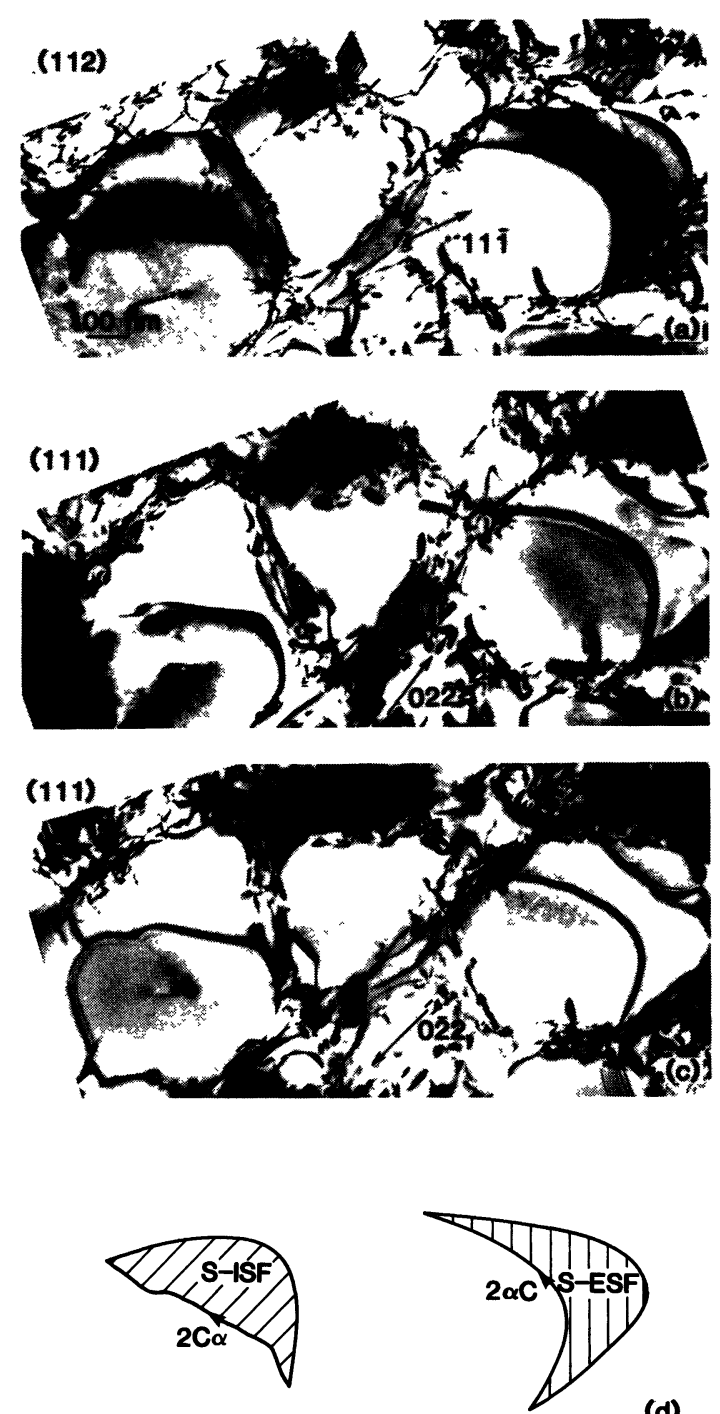

(d)

Fig. 5. - Configuration de cisaillement de précipités $\gamma^{\prime}$ dans l'alliage AM1 deformé en traction dynamique a $950^{\circ} \mathrm{C}$. (a) micrographie en champ clair, $g=111$; (b) microphie en champ clair, $g=02 \overline{2}$, (c) micrographie en champ clair, $g=0 \overline{2} 2$; (d) représentation schématique de la configuration, S-ISF (SSF intrinsèque), $S$ ESF (SSF extrinsèque).

[Shearing configuration of $\gamma^{\prime}$ precipitates in the alloy AM1 deformed in dynamical tension at $950^{\circ} \mathrm{C}$ : (a) bright-field micrograph in $g=11 \overline{1}$; (b) bright-field micrograph in $g=022$; (c) bright-field micrograph in $g=0 \overline{2} 2$; (d) scheme of the configuration, S-ESF (superlattice extrinsic stacking fault).] 
3.5 Fluage COMPRESSION À TEMPÉRATURE INTERMÉdiAire [13]. - Les observations montrent que les précipités sont cisaillés, comme pour l'essai précédent, par des configurations faisant intervenir un mélange de SSF extrinsèques et intrinsèques mais dans ce cas, la proportion de $S$ ESF apparait supérieure. Le processus de cisaillement fait également intervenir des dislocations de matrice $\mathrm{a} / 2<110>$ qui laissent à l'interface une Shockley de nature différente de celles qui cisaillent le précipité.

Les figures 6 et 7 montrent quelques exemples de cisaillement des précipités dans les plans $\{111\}$ inclinés.
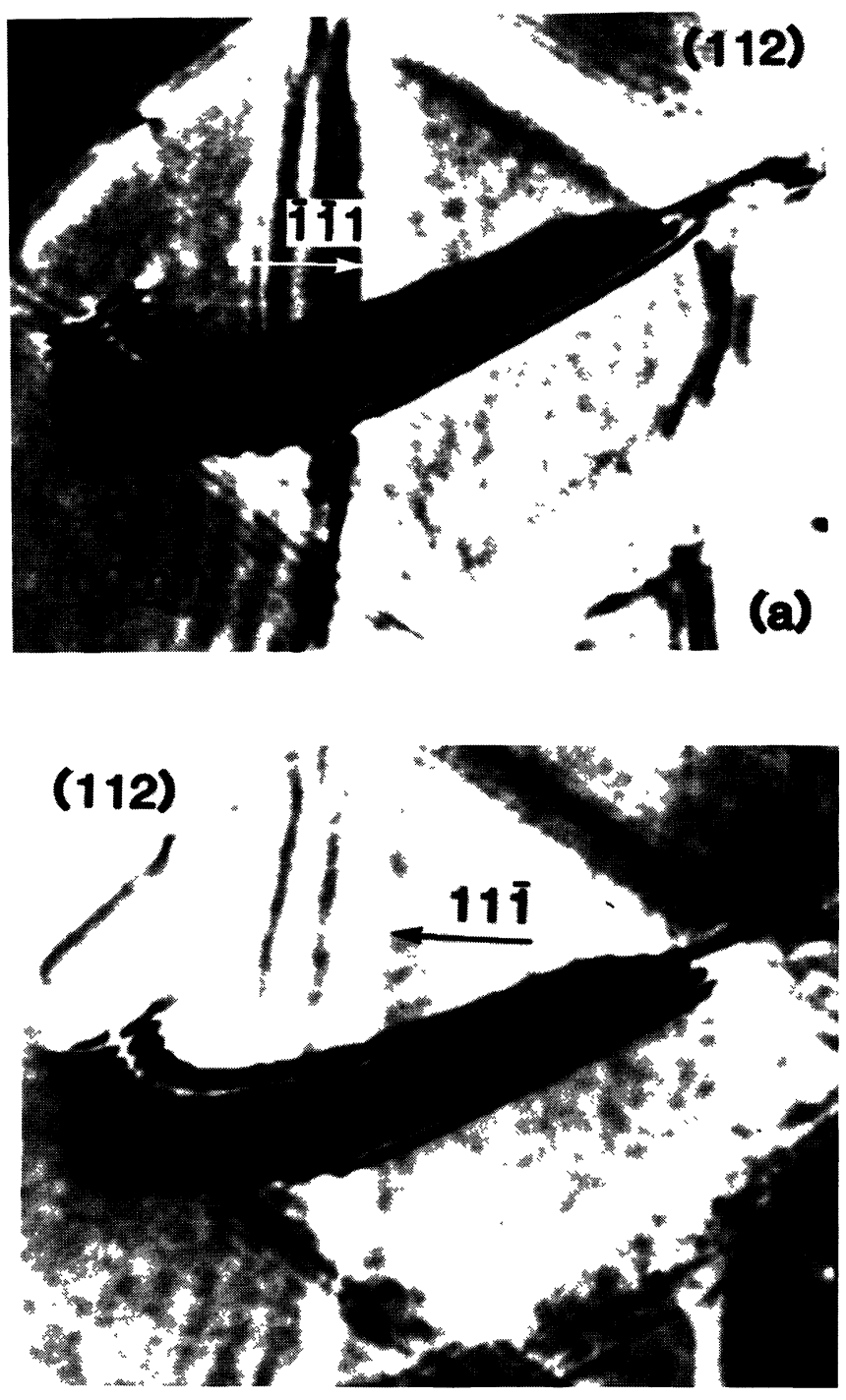

Fig. 6. - Configuration de cisaillement d'un précipité $\gamma^{\prime}$ dans un plan $\{111\}$ incliné dans l'alliage AM3 déformé en fluage compression à $750^{\circ} \mathrm{C}$. (a) micrographie en champ clair, $g=\overline{1} \overline{1} 1$; (b) micrographie en champ clair, $g=11 \overline{1}$.

[Shearing configuration of a $\gamma^{\prime}$ precipitate in an inclined $\{111\}$ plane in the alloy AM3 after a compression creep test at $750^{\circ} \mathrm{C}$ : (a) bright field micrograph in $g=\overline{1} 1 \overline{1}$; (b) bright field micrograph in $g=11 \overline{1}$.] 
La figure 6 présente deux micrographies en champ clair prises avec $+g$ et $-g$ d'une double faute dans le plan incliné $\beta$. L'analyse de la nature des défauts a été faite en utilisant la présence d'une frange blanche extérieure pour $g \cdot R=1 / 3$ [10] et montre que la première faute est intrinsèque (S-ISF). La frange blanche extérieure est présente pour le défaut intrinsèque en figure $6 a$ tandis que celle pour le défaut extrinsèque est présente en figure $6 \mathrm{~b}$.

La figure 7 montre deux micrographies en champ clair de quatre défauts situés dans le plan incliné $\delta$. Trois des défauts sont intrinsèques et présentent un frange blanche extérieure en figure 7a. L'autre défaut est extrinsèque et présente une frange blanche extérieure en figure $7 \mathrm{~b}$.

\section{Résumé des résultats expérimentaux.}

Mis à part le fluage traction à $950^{\circ} \mathrm{C}$, les configurations de cisaillement des précipités font toutes intervenir des superdéfauts d'empilement.

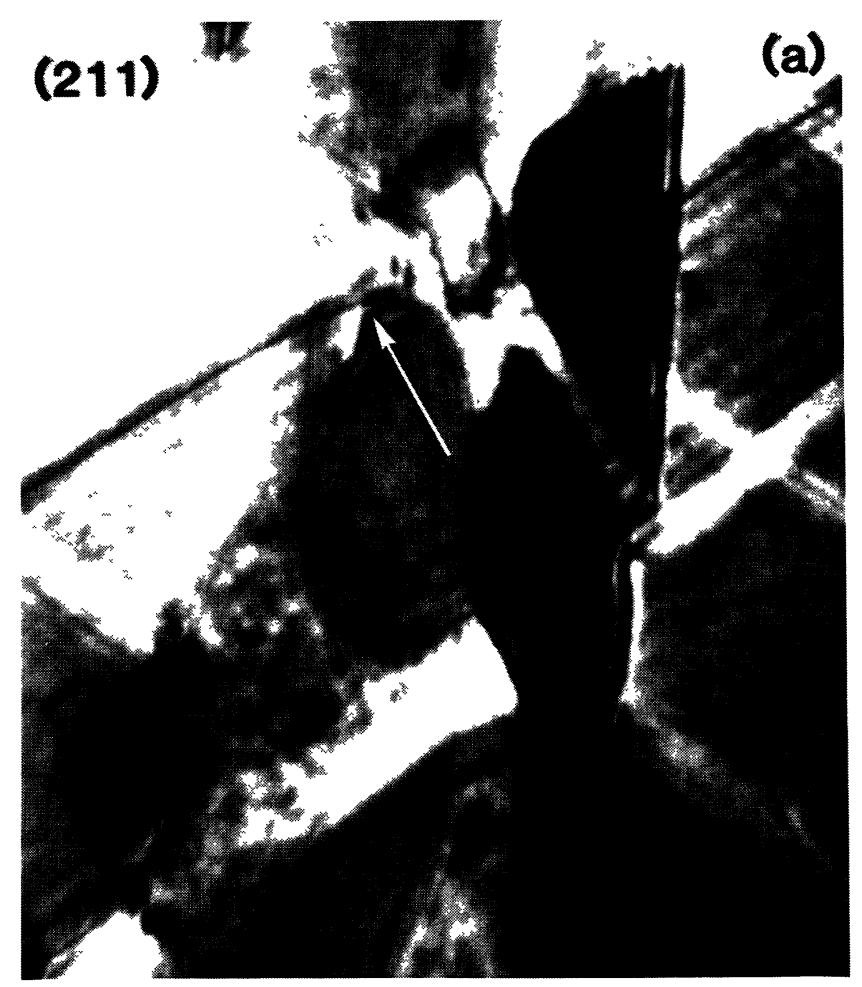

Fig. 7. - Configuration de cisaillement de précipités $\gamma^{\prime}$ dans un plan $\{111\}$ incliné dans l'alliage AM3 déformé en fluage compression à $750^{\circ} \mathrm{C}$. (a) micrographie en champ clair, $g=1 \overline{1} \overline{1}$; (b) micrographie en champ clair, $g=111$.

[Shearing configuration of $\gamma$ 'precipitates in an inclined $\{111\}$ plane in the alloy AM3 after a compression creep test at $750^{\circ} \mathrm{C}$. (a) Bright field micrograph in $g=11 \overline{1}$; (b) Bright field micrograph in $g=\overline{1} 11$.] 


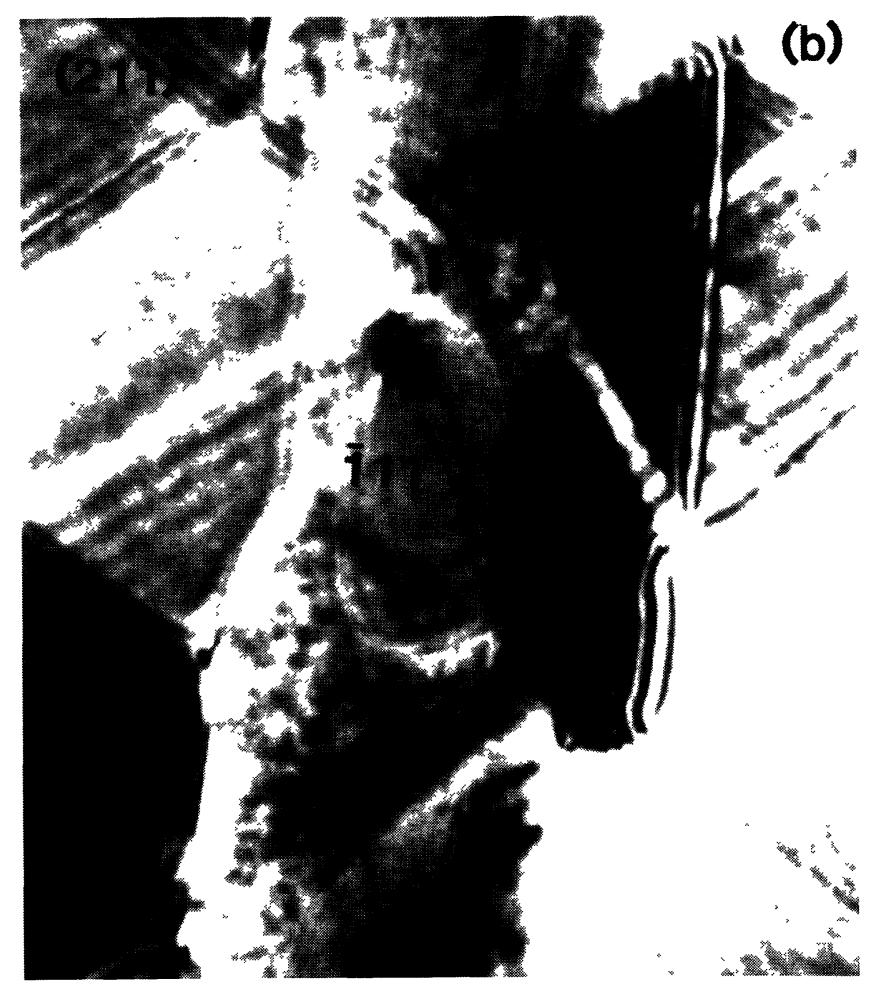

Fig. 7. - (continued)

- La nature des défauts dépend de l'essai mécanique effectué:

- en compression dynamique à $950^{\circ} \mathrm{C}$, les SSF simples sont essentiellement de nature extrinsèque (S-ESF);

- en fluage traction à température intermédiaire, les SSF simples sont essentiellement de nature intrinsèque (S-ISF);

- en traction dynamique à $950^{\circ} \mathrm{C}$, il y a un mélange de S-ISF et S-ESF avec une plus forte proportion apparente de S-ISF;

- en fluage compression à température intermédiaire, il y a un mélange de S-ESF et S-ISF avec une plus forte proportion apparente de S-ESF.

- Il y a une asymétrie entre les essais de fluage traction et compression à température intermédiaire (axe <001>) : les superalliages sont plus résistants en traction qu'en compression (vitesse de déformation plus rapide en compression qu'en traction).

- Les configurations de cisaillement font intervenir des dislocations de matrice $a / 2<110>$ qui laissent à l'interface une dislocation de Shockley de nature différente de celles qui cisaillent les précipités à la fois pour la formation de S-ISF et S-ESF.

- La dislocation $a / 3<112>$ qui cisaille le précipité est dissociée à l'échelle du champ faible en deux dislocations identiques $a / 6<112>$ séparées par un défaut complexe qui a été trouvée d'énergie voisine dans les deux cas (formation de S-ISF et de S-ESF).

-Il n'y a aucune différence entre les deux alliages AM1 et AM3 en ce qui concerne les configurations de cisaillement. Les valeurs d'énergie de défaut complexe sont du même ordre. 


\section{Interprétation et discussion.}

Les observations montrent que, excepté le cas du fluage traction à haute température, toutes les configurations de cisaillement rencontrées pour les autres essais font intervenir des superdéfauts d'empilement (S-ISF et S-ESF). L'analyse détaillée de ces configurations a remis en question le mécanisme proposé par Kear et al. depuis plus d'une quinzaine d'années [4] car :

- les configurations de cisaillement font intervenir des dislocations de matrice $a / 2<110>$ et non pas $a / 2<112>$ comme l'a proposé Kear;

- il existe à l'interface des Shockley de nature différente de celles qui cisaillent les précipités.

- les dislocations de matrice, qui font partie des configurations de cisaillement, se déplacent inépendamment les unes des autres et dans certains cas une seule dislocation de matrice est initiatrice du cisaillement (Fig. 4);

- la formation de la dislocation de Shcokley $\alpha \mathrm{C}$ laissée à l'interface dans la configuration de la figure 2 ne peut pas être expliqué par des réactions entre dislocations.

Ces faits ont conduit à proposer un mécanisme original de cisaillement des précipités à partir d'ue seule dislocation de matrice $a / 2<110>$ et faisant intervenir la nucléation d'une Shockley. Ce mécanisme est décrit en figure 8 : une dislocation $a / 2<110>$ arrive à l'interface et pénètre dans le précipité créant une paroi d'antiphase (APB) (Fig. 8a). Une dislocation de Shockley nuclée (Fig. 8b) transformant l'APB en superdéfaut d'empilement (S-ISF si la Shockley est de nature intrinsèque ou S-ESF si elle est extrinsèque). Un tel processus est similaire à celui proposé théoriquement par Kear et al. [15], où la nucléation se produit à l'interface $\gamma / \gamma^{\prime}$. Puis la dislocation $\mathrm{BD}$ poursuit sa progression, remplacée dans le précipité par une paire de Shockley $\mathrm{B} \alpha$ (ou $\alpha \mathrm{D})$. Elle laisse une Shockley $\mathrm{C} \alpha$ (ou $\alpha \mathrm{C}$ ) à l'interface (Fig. 8c). Cette Shockley peut agir comme piège pour d'autres dislocations : si une dislocation $\mathrm{BC}$ arrive à l'interface, la partielle $\alpha \mathrm{C}$ de la dislocation va réagir avec la Shockley d'interface et la configuration de la figure 1 est obtenue. Si une seconde dislocation $\mathrm{BD}$ se présente à l'interface, on obtient la configuration de la figure 2 . Un tri des dislocations peut ainsi avoir lieu en fonction de la nature de la Shockley laissée à l'interface.
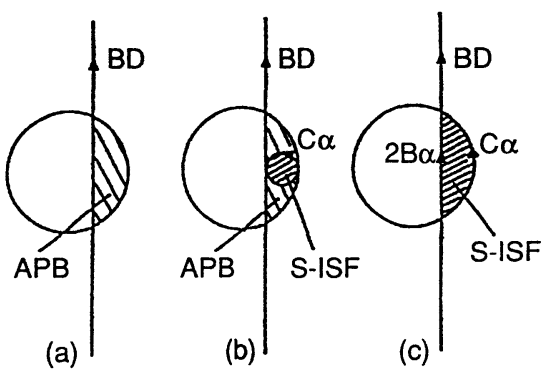

Fig. 8. - Mécanisme de cisaillement d'un précipité $\gamma^{\prime}$ par une dislocation de matrice parfaite. [Shearing mechanism of a $\gamma^{\prime}$ precipitate by a perfect matrix dislocation.]

La présence de S-ESF ne peut-être expliquée par notre mécanisme en considérant le franchissement des précipités $\gamma^{\prime}$ par des dislocations de matrice non dissociées. En effet, il est généralement admis que la nucléation d'une Shockley extrinsèque coûte plus cher en énergie que celle d'une Shockley intrinsèque.

Courbon [16] a pu contourner cette difficulté en faisant intervenir la loi de Schmidt qui favorise ou au contraire s'oppose au développement d'une boucle de Shockley (aux erreurs expérimentales 
près, il est plus difficile de rendre compte du mélange S-ISF, S-ESF dans le cadre de ce type d'explication).

Néanmoins, la présence de S-ESF, ainsi que la nature des différents défauts observés suivant les essais peut être expliquée à partir du mécanisme proposé si l'on considère que les dislocations de matrice $a / 2<110>$ sont dissociées. Il convient alors de tenir compte de deux effets dont la résultante peut conduire à la présence de S-ISF, de S-ESF ou bien du mélange des deux:

- une barrière d'énergie au franchissement de l'interface par une partielle de Shockley;

- l'effet de la force résolue dans le plan $\{111\}$ de glissement de la dislocation de matrice (venant de la contrainte appliquée) sur la largeur de dissociation de la dislocation de matrice.

Il a été montré $[17,18]$ que pour l'orientation $<001>$, une contrainte de compression augmente la largeur de dissociation tandis qu'une contrainte de traction la diminue. Ceci nous amène à compléter le mécanisme de cisaillement des précipités pour une dislocation de matrice dissociée dans les deux cas (S-ESF et S-ISF). Ce modèle est décrit en figure 9 pour le cas extrinsèque et en figure 10 pour l'intrinsèque.

En compression dynamique, la contrainte appliquée augmente la largeur de dissociation (force plus grande sur la partielle de tête) (Fig. 9a), de telle façon que lorsque la dislocation arrive à l'interface, elle pénètre dans le précipité sans constriction (Fig. 9b) et la nucléation d'une Shockley sur la deuxième partielle (Fig. 9c) transforme l'APB en S-ESF (Fig. 9d).

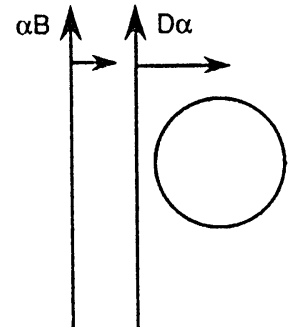

(a)

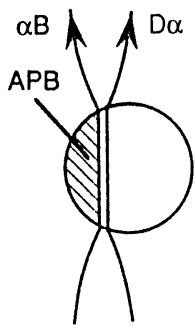

(b)

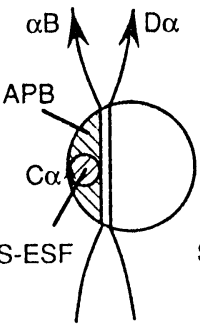

(c)

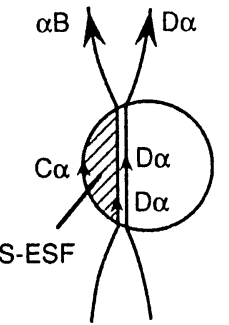

(d)

Fig. 9. - Mécanisme de cisaillement d'un précipité $\gamma^{\prime}$ par une dislocation de matrice dissociée (cas extrinsèque).

[Shearing mechansim of a $\gamma^{\prime}$ precipitate by a dissociated matrix dislocation (extrinsic case).]

En fluage traction, c'est l'inverse (Fig. 10a): la contrainte appliquée diminue la largeur de dissociation (force plus grande sur la partielle de queue) et une inversion des partielles se produit quand la dislocation pénètre dans le précipité (Fig. 10b). Dans ce cas, la nucléation d'une Shockley sur la deuxième partielle (Fig. 10c) conduit à la formation d'une S-ISF (Fig. 10d).

En ce qui concerne la traction dynamique, le mélange de défauts peut s'expliquer par le fait que en présence d'une forte contrainte appliquée et à grande vitesse de déformation, la contrainte résolue est assez élevée dans certains cas pour que la première partielle pénètre dans le précipité bien que la largeur de dissociation soit diminuée et ceci conduit à la formation de S-ESF, l'énergie fournie étant suffisante pour nucléer des Shockleys extrinsèques.

Pour le fluage compression, bien que la largeur de dissociation soit augmentée, il peut arriver que la contrainte résolue ne soit pas suffisamment élevée pour que la première partielle pénètre dans le précipité. On se retrouve dans le cas de la figure 10 conduisant à la formation de S-ISF, d'où mélange des défauts. 


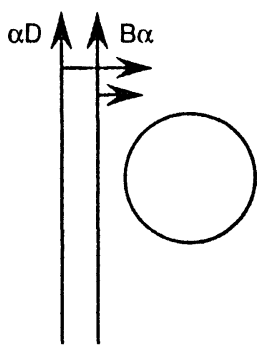

(a)

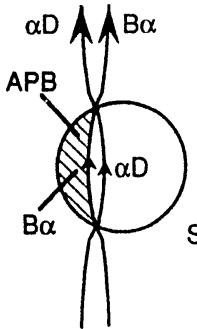

(b)

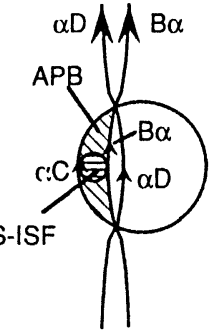

(c)

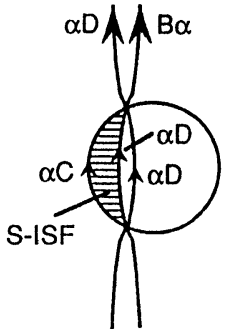

(d)

Fig. 10. - Mécanisme de cisaillement d'un précipité $\gamma^{\prime}$ par une dislocation de matrice dissociée (cas intrinsèque).

[Shearing mechanism of a $\gamma^{\prime}$ precipitate by a dissociated matrix dislocation (intrinsic case).]

Un tel mécanisme nous permet de fournir une explication originale de la dépendance du cisaillement des précipités avec la température en fluage primaire :

- à haute température $\left(>850^{\circ} \mathrm{C}\right)$, les précipités sont cisaillés par des paires de dislocations $a / 2$ $<110>$ couplées par une APB de haute énergie;

- à température intermédiare $\left(\approx 760^{\circ} \mathrm{C}\right)$, les précipités sont cisaillés par des dislocations $a / 3$ $<112>$ séparées par des superdéfauts d'empilement (S-ISF/S-ESF).

Pour expliquer ce changement de mode avec la température, plusieurs hypothèses ont été avancées: soit un changement d'énergie de défaut d'empilement de la matrice (SFE) ou une variation de l'énergie d'A.PB [19] et plus récemment l'existence de la montée des dislocations sur les dislocations bloquées à i’interface [6].

Les observations montrent que le phénomène n'est pas lié à une variation d'énergie d'APB car les essais de compression dynamique et traction dynamique à haute température que nous venons de décrire font intervenir des processus de cisaillement par des superdéfauts d'empilement à une température où l'on ne devrait pas les observer s'il y avait abaissement de l'énergie de paroi d'antiphase.

Les résultats présentés montrent qu'en fluage à $950^{\circ} \mathrm{C}$, la coalescence des précipités joue un rôle important, interdisant toute inversion des partielles de Shockley par effet chimique [14] : en effet, les dislocations sont absorbées par le précipité en cours de coalescence. Ainsi la seule possibilité pour créer des superdéfauts d'empilement serait de nucléer une Schockley extrinsèque sur la deuxième partielle, processus sans doute trop coûteux en énergie compte tenu de la faible contrainte appliquée lors de l'essai.

L'effet de la montée des dislocations bloquées à l'interface $\gamma / \gamma^{\prime}$, par la formation de constrictions, favorise le franchissement par superpartielles séparées par une paroi d'antiphase (nucléation et propagation des shockley rendues difficiles).

La dissymétrie observée à l'échelle macroscopique en début de fluage (moins grande résistance à température intermédiaire en compression qu'en traction pour une même déformation plastique) peut aussi se comprendre à l'aide d'un tel modèle : en traction, le cisaillement nécessite la recombinaison et l'inversion des partielles.

\section{Conclusions.}

La microscopie électronique en champ faible a été utilisée pour étudier les processus de cisaillement des précipités dans des monocristaux de superalliages base nickel. Les essais révèlent une 
asymétrie traction/compression : en compression dynamique, les configurations de cisaillement font intervenir des S-ESF et en fluage traction, elles font intervenir des S-ISF. Il y a un mélange de S-ISF et S-ESF en traction dynamique et en fluage compression.

A partir de ces résultats, nous proposons un modèle original de cisaillement des précipités basé sur des mécanismes de franchissement de l'interface par une simple dislocation de matrice $a / 2$ $<110>$ dissociée. le modèle se comprend simplement en considérant le bilan de deux effets :

- une barrière d'énergie au franchissement de l'interface par des Shockley de matrice élémentaires;

- Un effet de la contrainte appliquée sur la largeur de dissociation des dislocations de matrice.

Il a également pu être montré comment intervenait la coalescence des précipités $\gamma^{\prime}$. La dissymétrie constatée va dans le même sens que celle connue du comportement à l'échelle macroscopique.

Enfin le mécanisme permet :

- de prévoir le comportement des dislocations pour d'autres orientations que $<001>$ [15];

- d'expliquer la dépendance des processus de cisaillement avec la température.

Il semble que les résultats obtenus puissent s'appliquer à l'ensemble de cette catégorie de superalliages base nickel.

\section{References}

[1] LeVERANT G. R. et KeAR B. H., Metall. Trans. 1 (1970) 491.

[2] LEVERANT G. R., KeAR B. H. et OBlaK J. M., Metall. Trans. 4 (1973) 355.

[3] CARON P. et KHAN T., Mater. Sci. Eng. 61 (1983) 173.

[4] KeAR B. H. et OblaK J. M., J. Phys. Colloq. France 35 (1974) C7-35.

[5] Huis INT VELD A. J., BOOM G., BRONSVELD P. M. et DE HOSSON J. Th. M., Scripta Metall. 19 (1985) 1123.

[6 DECAMPs B. et CondaT M., J. Microsc. Spectrosc. Electron. 11 (1986) 141.

[7] CONDAT M. et DÉCAMPS B., Scripta Metall. 21 (1987) 607.

[8] Caron P., Khan T. et Veyssière P., Philos. Mag. A. 57 (1988) 859.

[9] Cockayne D. J. H., RaY I. L. F. et Whelan M. J., Philos. Mag. 20 (1969) 1265.

[10] HeAd A. K., Humble P., Clarebrough L. M., MORTON A. J. et FoRwOOD C. T., Computed Electron Micrographs and Defects Identification (North-Holland, Amsterdam, 1973).

[11] Bilby B. H., Bullough R. et SMITH E., Proc. Roy Soc. A231 (1955) 263.

[12] Thompson N., Proc. Phys. Soc. B66 (1953) 481.

[13] DÉCAMPS B., MorTon A. J. et CONDAT M., Philos. Mag. A. 64 (1991) 641.

[14] DécamPs B. et CondaT M., Phys. Stat. Sol (a) 109 (1988) K15.

[15] KeAR B. H., Giamei A. F., Silcock J. M. et HAM R. K., Scripta Metall. 2 (1968) 287.

[16] COURBON J., Thèse d'Université INPG, Grenoble (1990).

[17] ClaRebrough L. M., ForwOOD C. T. et MORTON A. J., Crystal Lattice Defects 4 (1973) 179.

[18] ShaH D. M. et DuHL D. N., Proc. 5th Intern. Symp. on Superalloys, Metallurgical Society AIME (1984) p. 105.

[19] KEAR B. H., Order-Disorder Transformations in Alloys, Verlimont Ed. (Springer-Verlag Berlin Heidelberg New-York, 1974) p. 440. 